Honam Mathematical J. 35 (2013), No. 4, pp. 809-817

http://dx.doi.org/10.5831/HMJ.2013.35.4.809

\title{
STRUCTURES OF HYPERHOLOMORPHIC FUNCTIONS ON DUAL QUATERNION NUMBERS
}

\author{
Hyun Sook Jung, Su Jin Ha, Kwang Ho Lee, Su Mi Lim \\ AND KWANG Ho SHON*
}

\begin{abstract}
We research properties of a corresponding Cauchy theorem of hyperholomorphic functions in an open set of product complex spaces, in the sense of complex Clifford analysis.
\end{abstract}

\section{Introduction}

Theory of holomorphic functions of several complex variables was developed by a constructive method of complex analysis. Weil [14] generalized the Cauchy integral formula to polynomial polyhedra in $\mathbb{C}^{n}$. Oka [6-13] solved the so-called fundamental problems, that is, Cousin problem and Levi problem et al. Likewise in the theory of holomorphic functions of several complex variables, investigations of hyperholomorphic functions, regular functions, monogenic functions, biregular functions and their natural domains of existence are rooted in abstract versions of complex Clifford analysis and their applications. In practice, we require $n$ solutions of polynomial equations having the form

$$
c_{0} z^{n}+c_{1} z^{n-1}+c_{2} z^{n-2}+\cdots+c_{n-1} z+c_{n}=0,
$$

where $c_{0} \neq 0, c_{1}, \cdots, c_{n}$ are given complex numbers and $n$ is a positive integer called the degree of the equation. The fundamental theorem of algebra does not hold in the case of complex Clifford analysis.

Received November 22, 2013. Accepted December 4, 2013.

2010 Mathematics Subject Classification. 32A99, 30G35, 11E88.

Key words and phrases. Hyperholomorphic function, quaternion number, dual number system, Clifford analysis, complex differential equation.

${ }^{*}$ Corresponding author 


\section{Preliminaries}

For the real space $\mathbb{R}^{4}$, the non-commutative extension of the complex numbers was discovered by Hamilton [3], and was called the quaternion in Clifford analysis. Futer [1] gave a definition of regular quaternion function in $\mathbb{R}^{4}$ and developed theory of quaternion functions in 1935 . Recently, theory of quaternion analysis have been applied in physics (see $[2])$.

The field $\mathcal{T}$ of quaternions

$$
z=\sum_{j=o}^{3} e_{j} x_{j}\left(x_{0}, x_{1}, x_{2}, x_{3} \in \mathbb{R}\right)
$$

is a four dimensional non-commutative $\mathbb{R}$-field of real numbers such that its four base elements $e_{0}=i d ., e_{1}, e_{2}$ and $e_{3}$ satisfy the followings:

$$
\begin{array}{ll}
e_{j}^{2}=-1(j=1,2,3), & e_{1} e_{2}=e_{3}=-e_{2} e_{1}, \\
e_{2} e_{3}=e_{1}=-e_{3} e_{2}, & e_{3} e_{1}=e_{2}=-e_{1} e_{3} .
\end{array}
$$

Identifying the element $e_{1}$ with the imaginary unit $\sqrt{-1}$ in the $\mathbb{C}$-field of complex numbers, we have a quaternion that $z$ is regarded as

$$
z=z_{1}+z_{2} e_{2} \in \mathcal{T}
$$

where $z_{1}=x_{0}+e_{1} x_{1}, z_{2}=x_{2}+e_{1} x_{3} \in \mathbb{C}$. Thus, we identify $\mathcal{T}$ with $\mathbb{C}^{2}$. We define the quaternion multiplication of two quaternion numbers $z=z_{1}+z_{2} e_{2}$ and $w=w_{1}+w_{2} e_{2} \in \mathcal{T}$, by where $\overline{z_{1}}=x_{1}-e_{1} x_{2}, \overline{z_{2}}=$ $x_{2}-e_{1} x_{3} \in \mathbb{C}$. For $z=z_{1}+z_{2} e_{2}$, the quaternion conjugate $z^{*}$, the absolute value $|z|$ of $z$ and the inverse $z^{-1}$ of $z$ in $\mathcal{T}$ are defined by the followings:

$$
z^{*}=\overline{z_{1}}-z_{2} e_{2},|z|=\sqrt{\left|z_{1}\right|^{2}+\left|z_{2}\right|^{2}}, z^{-1}=\frac{z^{*}}{|z|^{2}}(z \neq 0) .
$$

We use the following quaternion differential operators:

$$
\begin{aligned}
& D_{z}:=\frac{\partial}{\partial z_{1}}-e_{2} \frac{\partial}{\partial \overline{z_{2}}}=\frac{1}{2}\left(\frac{\partial}{\partial x_{0}}-e_{1} \frac{\partial}{\partial x_{1}}-e_{2} \frac{\partial}{\partial x_{2}}+e_{3} \frac{\partial}{\partial x_{3}}\right), \\
& D_{z}^{*}=\frac{\partial}{\partial \overline{z_{1}}}+e_{2} \frac{\partial}{\partial \overline{z_{2}}}=\frac{1}{2}\left(\frac{\partial}{\partial x_{0}}+e_{1} \frac{\partial}{\partial x_{1}}+e_{2} \frac{\partial}{\partial x_{2}}-e_{3} \frac{\partial}{\partial x_{3}}\right),
\end{aligned}
$$


where $\frac{\partial}{\partial z_{1}}, \frac{\partial}{\partial z_{1}}, \frac{\partial}{\partial z_{2}}$ and $\frac{\partial}{\partial z_{2}}$ are usual differential operators. And, we have

$$
\begin{aligned}
\frac{\partial}{\partial z_{1}} e_{2} & =\frac{1}{2}\left(\frac{\partial}{\partial x_{0}}-e_{1} \frac{\partial}{\partial x_{1}}\right) e_{2}=\frac{1}{2}\left(e_{2} \frac{\partial}{\partial x_{0}}-e_{1} e_{2} \frac{\partial}{\partial x_{1}}\right) \\
& =\frac{1}{2}\left(e_{2} \frac{\partial}{\partial x_{0}}+e_{2} e_{1} \frac{\partial}{\partial x_{1}}\right)=e_{2} \frac{\partial}{\partial \bar{z}_{1}}, \\
\frac{\partial}{\partial \bar{z}_{1}} e_{2} & =\frac{1}{2}\left(\frac{\partial}{\partial x_{0}}+e_{1} \frac{\partial}{\partial x_{1}}\right) e_{2}=\frac{1}{2}\left(e_{2} \frac{\partial}{\partial x_{0}}+e_{1} e_{2} \frac{\partial}{\partial x_{1}}\right) \\
& =\frac{1}{2}\left(e_{2} \frac{\partial}{\partial x_{0}}-e_{2} e_{1} \frac{\partial}{\partial x_{1}}\right)=e_{2} \frac{\partial}{\partial z_{1}} .
\end{aligned}
$$

The operator

$$
\begin{aligned}
D_{z} D_{z}^{*} & =\left(\frac{\partial}{\partial z_{1}}-e_{2} \frac{\partial}{\partial \overline{z_{2}}}\right)\left(\frac{\partial}{\partial \overline{z_{1}}}+e_{2} \frac{\partial}{\partial \overline{z_{2}}}\right) \\
& =\frac{1}{4}\left(\frac{\partial^{2}}{\partial x_{1}^{2}}+\frac{\partial^{2}}{\partial x_{2}^{2}}+\frac{\partial^{2}}{\partial x_{3}^{2}}+\frac{\partial^{2}}{\partial x_{4}^{2}}\right)=\Delta_{x} .
\end{aligned}
$$

Let $D$ be an open set in $\mathbb{C}^{2}$ and $f(z)=f_{1}(z)+f_{2} e_{2}$ be a function defined on $D$ with values in $\mathcal{T}$, where $z=\left(z_{1}, z_{2}\right)$ and $f_{1}(z)$ and $f_{2}(z)$ are complex-valued functions on $D$.

Definition 1. Let $D$ be an open set in $\mathbb{C}^{2}$. A function $f(z)=f_{1}(z)+$ $f_{2}(z) e_{2}$ is said to be $L(R)$-hyperholomorphic on $D$, if

(1) $f_{1}(z)$ and $f_{2}(z)$ are continuously differentiable on $D$,

(2) $\frac{\partial}{\partial z^{*}} f=0\left(f \frac{\partial}{\partial z^{*}}=0\right)$ on $D$.

The above equations (2) of the definition 1 operate to $f$ as follows:

$$
\begin{aligned}
& D_{z}^{*} f=\left(\frac{\partial}{\partial \overline{z_{1}}}+e_{2} \frac{\partial}{\partial \overline{z_{2}}}\right)\left(f_{1}+f_{2} e_{2}\right)=\left(\frac{\partial f_{1}}{\partial \overline{z_{1}}}-\frac{\partial \overline{f_{2}}}{\partial z_{2}}\right)+\left(\frac{\partial f_{2}}{\partial \overline{z_{1}}}+\frac{\partial \overline{f_{1}}}{\partial z_{2}}\right) e_{2}, \\
& f D_{z}^{*}=\left(f_{1}+f_{2} e_{2}\right)\left(\frac{\partial}{\partial \overline{z_{1}}}+e_{2} \frac{\partial}{\partial \overline{z_{2}}}\right)=\left(\frac{\partial f_{1}}{\partial \overline{z_{1}}}-\frac{\partial f_{2}}{\partial \overline{z_{2}}}\right)+\left(\frac{\partial f_{2}}{\partial z_{1}}+\frac{\partial f_{1}}{\partial z_{2}}\right) e_{2} .
\end{aligned}
$$

The above equation (2) of the definition 1 for $L$-hyperholomorphic function $f(z)$ is equivalent to the following corresponding Cauchy-Riemann system in $\mathcal{T}$ :

$$
\frac{\partial f_{1}}{\partial \bar{z}_{1}}=\frac{\partial \bar{f}_{2}}{\partial z_{2}}, \frac{\partial f_{2}}{\partial \bar{z}_{1}}=-\frac{\partial \bar{f}_{1}}{\partial z_{2}}
$$




\section{Dual quaternion}

The dual numbers extended the real numbers by adjoining one new element $\varepsilon$ with the property $\varepsilon^{2}=0$. Every dual number has the form $z=x+\varepsilon y$ with $x$ and $y$ uniquely determined real numbers. And the conjugate dual number $z^{*}$ of $z$ is defined by $z^{*}=x-\varepsilon y$ and we obtain $|z|^{2}=x^{2}$. If we use matrices, dual numbers can be represented as

$$
\varepsilon=\left(\begin{array}{ll}
0 & 1 \\
0 & 0
\end{array}\right), z=x+\varepsilon y=\left(\begin{array}{ll}
x & y \\
0 & x
\end{array}\right) .
$$

For the real polynomial, we have

$$
\begin{aligned}
P(x)= & p_{n} x^{n}+p_{n-1} x^{n-1}+\cdots+p_{1} x+p_{0}, \\
P(x+\varepsilon y)= & p_{n}(x+\varepsilon y)^{n}+p_{n-1}(x+\varepsilon y)^{n-1}+\cdots+p_{1}(x+\varepsilon y)+p_{0} \\
= & p_{n} x^{n}+\varepsilon n p_{n} x^{n-1} y+p_{n-1} x^{n-1}+\varepsilon(n-1) p_{n-1} x^{n-2} y \\
& \quad+\cdots+p_{1} x+\varepsilon p_{1} y+p_{0} \\
= & P(x)+\varepsilon y P^{\prime}(x) .
\end{aligned}
$$

For a Taylor series of a holomorphic function $f(a+\varepsilon b)$, we have

$$
f(x+\varepsilon y)=\sum_{n=0}^{\infty} \frac{f^{(n)}(x)}{n !}(\varepsilon y)^{n}=f(x)+\varepsilon y f^{\prime}(x) .
$$

Hence, we have

$$
\exp (\varepsilon y)=\sum_{n=0}^{\infty} \frac{(\varepsilon y)^{n}}{n !}=1+\varepsilon y
$$

Thus,

$$
(x+\varepsilon y)^{c+\varepsilon d}=x^{c}+\varepsilon\left[y\left(c x^{c-1}\right)+d\left(x^{c} \ln x\right)\right] .
$$

Therefore, the dual numbers are elements of the 2-dimensional real algebra

$$
D=\left\{z=x+\varepsilon y \mid x, y \in \mathbb{R}, \varepsilon^{2}=0\right\}
$$

generated by 1 and $\varepsilon$. The dual quaternion $z=\sum_{j=0}^{3} e_{j} x_{j}+\varepsilon \sum_{j=0}^{3} e_{j} y_{j}$ is written as

$$
z=a+\varepsilon b .
$$

The conjugation number $z^{*}$, the absolute value $|z|$ and the inverse $z^{-1}$ of $z=a+\varepsilon b$ are given by the followings:

$$
\begin{aligned}
& z^{*}=x_{0}-e_{1} x_{1}-e_{2} x_{2}-e_{3} x_{3}+\varepsilon\left(y_{0}-e_{1} y_{1}-e_{2} y_{2}-e_{3} y_{3}\right)=a^{*}+\varepsilon b^{*}, \\
& |z|^{2}=z z^{*}=\sum_{j=0}^{3} x_{j}{ }^{2}+2 \varepsilon \sum_{j=0}^{3} x_{j} y_{j}=\sum_{j=0}^{3} \zeta_{j}^{2}, z^{-1}=\frac{z^{*}}{|z|^{2}}(z \neq 0),
\end{aligned}
$$


where $\zeta_{j}=x_{j}+\varepsilon y_{j}(j=0,1,2,3), a^{*}$ and $b^{*}$ are conjugate numbers of $a$ and $b$, respectively.

We consider the following differential operators:

$$
\begin{aligned}
& D:=\frac{\partial}{\partial x_{0}}-e_{1} \frac{\partial}{\partial x_{1}}-e_{2} \frac{\partial}{\partial x_{2}}-e_{3} \frac{\partial}{\partial x_{3}}+\varepsilon\left(\frac{\partial}{\partial y_{0}}-e_{1} \frac{\partial}{\partial y_{1}}-e_{2} \frac{\partial}{\partial y_{2}}-e_{3} \frac{\partial}{\partial y_{3}}\right), \\
& D^{*}=\frac{\partial}{\partial x_{0}}+e_{1} \frac{\partial}{\partial x_{1}}+e_{2} \frac{\partial}{\partial x_{2}}+e_{3} \frac{\partial}{\partial x_{3}}+\varepsilon\left(\frac{\partial}{\partial y_{0}}+e_{1} \frac{\partial}{\partial y_{1}}+e_{2} \frac{\partial}{\partial y_{2}}+e_{3} \frac{\partial}{\partial y_{3}}\right) .
\end{aligned}
$$

Then, we have the following for dual quaternion operators:

$$
D D^{*}=D^{*} D=\sum_{j=0}^{3} \frac{\partial^{2}}{\partial x_{j}{ }^{2}}+2 \varepsilon\left(\sum_{j=0}^{3} \frac{\partial^{2}}{\partial x_{j} \partial y_{j}}\right)=\sum_{j=0}^{3} \frac{\partial^{2}}{\partial \tau_{j}{ }^{2}}=\Delta_{z},
$$

where

$$
\frac{\partial}{\partial \tau_{j}}=\frac{\partial}{\partial x_{j}}+\varepsilon \frac{\partial}{\partial y_{j}}(j=0,1,2,3)
$$

Definition 2. Let $\Omega$ be an open subset of $\mathbb{C}^{2} \times \mathbb{C}^{2}$. A function $F(z)=f(a)+\varepsilon g(b)=\sum_{j=0}^{3} e_{j} u_{j}(a)+\varepsilon\left(\sum_{j=0}^{3} e_{j} v_{j}(b)\right)$ is said to be hyperholomorphic on $\Omega$ if

(1) $u_{j}(a)$ and $v_{j}(b)(j=0,1,2,3)$ are continuously differentiable on $\Omega$,

(2) $D^{*} F(z)=0$ on $\Omega$.

Equation (2) of Definition 2 operates to $F(z)$ as follows:

$$
\begin{aligned}
D^{*} F & =\left(\sum_{j=0}^{3} e_{j} \frac{\partial}{\partial x_{j}}+\varepsilon \sum_{j=0}^{3} e_{j} \frac{\partial}{\partial y_{j}}\right)\left(\sum_{j=0}^{3} e_{j} u_{j}+\varepsilon \sum_{j=0}^{3} e_{j} v_{j}\right) \\
& =\left(\frac{\partial}{\partial x_{0}}+e_{1} \frac{\partial}{\partial x_{1}}+e_{2} \frac{\partial}{\partial x_{2}}+e_{3} \frac{\partial}{\partial x_{3}}+\varepsilon\left(\frac{\partial}{\partial y_{0}}+e_{1} \frac{\partial}{\partial y_{1}}+e_{2} \frac{\partial}{\partial y_{2}}\right.\right. \\
& \left.\left.+e_{3} \frac{\partial}{\partial x_{3}}\right)\right) \cdot\left(u_{0}+e_{1} u_{1}+e_{2} u_{2}+e_{3} u_{3}+\varepsilon\left(v_{0}+e_{1} v_{1}+e_{2} v_{2}+e_{3} v_{3}\right)\right) \\
& =\frac{\partial u_{0}}{\partial x_{0}}-\frac{\partial u_{1}}{\partial x_{1}}-\frac{\partial u_{2}}{\partial x_{2}}-\frac{\partial u_{3}}{\partial x_{3}}+e_{1}\left(\frac{\partial u_{1}}{\partial x_{0}}+\frac{\partial u_{0}}{\partial x_{1}}+\frac{\partial u_{3}}{\partial x_{2}}-\frac{\partial u_{2}}{\partial x_{3}}\right) \\
& +e_{2}\left(\frac{\partial u_{2}}{\partial x_{0}}-\frac{\partial u_{3}}{\partial x_{1}}+\frac{\partial u_{0}}{\partial x_{2}}+\frac{\partial u_{1}}{\partial x_{3}}\right)+e_{3}\left(\frac{\partial u_{3}}{\partial x_{0}}+\frac{\partial u_{2}}{\partial x_{1}}-\frac{\partial u_{1}}{\partial x_{2}}+\frac{\partial u_{0}}{\partial x_{3}}\right) \\
& +\varepsilon\left(\frac{\partial v_{0}}{\partial x_{0}}-\frac{\partial v_{1}}{\partial x_{1}}-\frac{\partial v_{2}}{\partial x_{2}}-\frac{\partial v_{3}}{\partial x_{3}}+\frac{\partial u_{0}}{\partial y_{0}}-\frac{\partial u_{1}}{\partial y_{1}}-\frac{\partial u_{2}}{\partial y_{2}}-\frac{\partial u_{3}}{\partial y_{3}}\right. \\
& +e_{1}\left(\frac{\partial v_{1}}{\partial x_{0}}+\frac{\partial v_{0}}{\partial x_{1}}+\frac{\partial v_{3}}{\partial x_{2}}-\frac{\partial v_{2}}{\partial x_{3}}+\frac{\partial u_{1}}{\partial y_{0}}+\frac{\partial u_{0}}{\partial y_{1}}+\frac{\partial u_{3}}{\partial y_{2}}-\frac{\partial u_{2}}{\partial y_{3}}\right)
\end{aligned}
$$




$$
\begin{aligned}
& +e_{2}\left(\frac{\partial v_{2}}{\partial x_{0}}-\frac{\partial v_{3}}{\partial x_{1}}+\frac{\partial v_{0}}{\partial x_{2}}+\frac{\partial v_{1}}{\partial x_{3}}+\frac{\partial u_{2}}{\partial y_{0}}-\frac{\partial u_{3}}{\partial y_{1}}+\frac{\partial u_{0}}{\partial y_{2}}+\frac{\partial u_{1}}{\partial y_{3}}\right) \\
& \left.+e_{3}\left(\frac{\partial v_{3}}{\partial x_{0}}+\frac{\partial v_{2}}{\partial x_{1}}-\frac{\partial v_{1}}{\partial x_{2}}+\frac{\partial v_{0}}{\partial x_{3}}+\frac{\partial u_{3}}{\partial y_{0}}+\frac{\partial u_{2}}{\partial y_{1}}-\frac{\partial u_{1}}{\partial y_{2}}+\frac{\partial u_{0}}{\partial y_{3}}\right)\right) .
\end{aligned}
$$

Therefore, the Equation (2) of Definition 2 for $F(z)$ is equivalent to the following system of equations:

$$
\begin{aligned}
& \frac{\partial u_{0}}{\partial x_{0}}=\frac{\partial u_{1}}{\partial x_{1}}+\frac{\partial u_{2}}{\partial x_{2}}+\frac{\partial u_{3}}{\partial x_{3}}, \frac{\partial u_{2}}{\partial x_{3}}=\frac{\partial u_{1}}{\partial x_{0}}+\frac{\partial u_{0}}{\partial x_{1}}+\frac{\partial u_{3}}{\partial x_{2}}, \\
& \frac{\partial u_{3}}{\partial x_{1}}=\frac{\partial u_{2}}{\partial x_{0}}+\frac{\partial u_{0}}{\partial x_{2}}+\frac{\partial u_{1}}{\partial x_{3}}, \frac{\partial u_{1}}{\partial x_{2}}=\frac{\partial u_{3}}{\partial x_{0}}+\frac{\partial u_{2}}{\partial x_{1}}+\frac{\partial u_{0}}{\partial x_{3}}, \\
& \frac{\partial v_{0}}{\partial x_{0}}+\frac{\partial u_{0}}{\partial y_{0}}=\frac{\partial v_{1}}{\partial x_{1}}+\frac{\partial v_{2}}{\partial x_{2}}+\frac{\partial v_{3}}{\partial x_{3}}+\frac{\partial u_{1}}{\partial y_{1}}+\frac{\partial u_{2}}{\partial y_{2}}+\frac{\partial u_{3}}{\partial y_{3}}, \\
& \frac{\partial v_{2}}{\partial x_{3}}+\frac{\partial u_{2}}{\partial y_{3}}=\frac{\partial v_{1}}{\partial x_{0}}+\frac{\partial v_{0}}{\partial x_{1}}+\frac{\partial v_{3}}{\partial x_{2}}+\frac{\partial u_{1}}{\partial y_{0}}+\frac{\partial u_{0}}{\partial y_{1}}+\frac{\partial u_{3}}{\partial y_{2}}, \\
& \frac{\partial u_{3}}{\partial y_{1}}+\frac{\partial v_{3}}{\partial x_{1}}=\frac{\partial v_{2}}{\partial x_{0}}+\frac{\partial v_{0}}{\partial x_{2}}+\frac{\partial v_{1}}{\partial x_{3}}+\frac{\partial u_{2}}{\partial y_{0}}+\frac{\partial u_{0}}{\partial y_{2}}+\frac{\partial u_{1}}{\partial y_{3}}, \\
& \frac{\partial v_{1}}{\partial x_{2}}+\frac{\partial u_{1}}{\partial y_{2}}=\frac{\partial v_{3}}{\partial x_{0}}+\frac{\partial v_{2}}{\partial x_{1}}+\frac{\partial v_{0}}{\partial x_{3}}+\frac{\partial u_{3}}{\partial y_{0}}+\frac{\partial u_{2}}{\partial y_{1}}+\frac{\partial u_{0}}{\partial y_{3}} .
\end{aligned}
$$

Now, we add the following condition of integrability:

$$
\begin{aligned}
& \frac{\partial v_{0}}{\partial x_{0}}=\frac{\partial v_{1}}{\partial x_{1}}+\frac{\partial v_{2}}{\partial x_{2}}+\frac{\partial v_{3}}{\partial x_{3}}, \frac{\partial v_{2}}{\partial x_{3}}=\frac{\partial v_{1}}{\partial x_{0}}+\frac{\partial v_{0}}{\partial x_{1}}+\frac{\partial v_{3}}{\partial x_{2}} \\
& \frac{\partial v_{3}}{\partial x_{1}}=\frac{\partial v_{2}}{\partial x_{0}}+\frac{\partial v_{0}}{\partial x_{2}}+\frac{\partial v_{1}}{\partial x_{3}}, \frac{\partial v_{1}}{\partial x_{2}}=\frac{\partial v_{3}}{\partial x_{0}}+\frac{\partial v_{2}}{\partial x_{1}}+\frac{\partial v_{0}}{\partial x_{3}} .
\end{aligned}
$$

We let

$$
d x y=d x_{0} \wedge d x_{1} \wedge d x_{2} \wedge d x_{3} \wedge d y_{0} \wedge d y_{1} \wedge d y_{2} \wedge d y_{3} .
$$

Theorem 3.1. Under the condition of integrability (3.2), let $F(z)$ be a hyperholomorphic function in an open set $\Omega$ of $\mathbb{C}^{2} \times \mathbb{C}^{2}$ and

$$
\kappa=d \hat{y}_{0}-e_{1} d \hat{y}_{1}+e_{2} d \hat{y}_{2}-e_{3} d \hat{y}_{3}+\varepsilon\left(\hat{x}_{0}-e_{1} d \hat{x}_{1}+e_{2} d \hat{x}_{2}-e_{3} d \hat{x}_{3}\right),
$$

where $d \hat{x}_{j}$ is the $d x_{j}$-removed form on $d x y$, and $\hat{d y_{j}}$ is the $d y_{j}$-removed form on $d x y(j=0,1,2,3)$. Then for any domain $G \subset \Omega$ with smooth distinguished boundary $b G$ of $\mathbb{C}^{2} \times \mathbb{C}^{2}$,

$$
\int_{b G} \kappa F(z)=0
$$


where $\kappa F(z)$ is the product of quaternion numbers of the form $\kappa$ on the function $F(z)$.

Proof. By the rule of the multiplication of quaternion numbers, we have

$$
\begin{aligned}
\kappa F(z)= & {\left[\hat{y}_{0}-e_{1} d \hat{y}_{1}+e_{2} d \hat{y}_{2}-e_{3} d \hat{y}_{3}+\varepsilon\left(d \hat{x}_{0}-e_{1} d \hat{x}_{1}+e_{2} d \hat{x}_{2}-e_{3} d \hat{x}_{3}\right)\right] } \\
& \cdot\left(\sum_{j=0}^{3} e_{j} u_{j}+\varepsilon \sum_{j=0}^{3} e_{j} v_{j}\right) \\
= & u_{o} d \hat{y}_{0}+e_{1} u_{1} d \hat{y}_{0}+e_{2} u_{2} d \hat{y}_{0}+e_{3} u_{3} d \hat{y}_{0}-e_{1} u_{0} d \hat{y}_{1}+u_{1} d \hat{y}_{1} \\
& -e_{3} u_{2} d \hat{y}_{1}+e_{2} u_{3} d \hat{y}_{1}+e_{2} u_{0} d \hat{y}_{2}-e_{3} u_{1} d \hat{y}_{2}-u_{2} d \hat{y}_{2}+e_{1} u_{3} d \hat{y}_{2} \\
& -e_{3} u_{0} d \hat{y}_{3}-e_{2} u_{1} d \hat{y}_{3}+e_{1} u_{2} d \hat{y}_{3}+u_{3} d \hat{y}_{3}+\varepsilon\left[v_{0} d \hat{y}_{0}+e_{1} v_{1} d \hat{y}_{0}\right. \\
& +e_{2} v_{2} d \hat{y}_{0}+e_{3} v_{3} d \hat{y}_{0}-e_{1} v_{0} d \hat{y}_{1}+v_{1} d \hat{y}_{1}-e_{3} v_{2} d \hat{y}_{1}+e_{2} v_{3} d \hat{y}_{1} \\
& +e_{2} v_{0} d \hat{y}_{2}-e_{3} v_{1} d \hat{y}_{2}-v_{2} d \hat{y}_{2}+e_{1} v_{3} d \hat{y}_{2}-e_{3} v_{0} d \hat{y}_{3}-e_{2} v_{1} d \hat{y}_{3} \\
& +e_{1} v_{2} d \hat{y}_{3}+v_{3} d \hat{y}_{3}+u_{0} d \hat{x}_{0}+e_{1} u_{1} d \hat{x}_{0}+e_{2} u_{2} d \hat{x}_{0}+e_{3} u_{3} d \hat{x}_{0} \\
& -e_{1} u_{0} d \hat{x}_{1}+u_{1} d \hat{x}_{1}-e_{3} u_{2} d \hat{x}_{1}+e_{2} u_{3} d \hat{x}_{1}+e_{2} u_{0} d \hat{x}_{2}-e_{3} u_{1} d \hat{x}_{2} \\
& \left.-u_{2} d \hat{x}_{2}+e_{1} u_{3} d \hat{x}_{2}-e_{3} u_{0} d \hat{x}_{3}-e_{2} u_{1} d \hat{x}_{3}+e_{1} u_{2} d \hat{x}_{3}+u_{3} d \hat{x}_{3}\right] .
\end{aligned}
$$

Therefore,

$$
\begin{aligned}
d(\kappa F) & =\left(\sum_{j=o}^{3} \frac{\partial}{\partial x_{j}} d x_{j}+\varepsilon \sum_{j=o}^{3} \frac{\partial}{\partial y_{j}} d y_{j}\right)(\kappa F) \\
& =\varepsilon\left[\left(\frac{\partial u_{0}}{\partial y_{0}}-\frac{\partial u_{1}}{\partial y_{1}}-\frac{\partial u_{2}}{\partial y_{2}}-\frac{\partial u_{3}}{\partial y_{3}}+\frac{\partial u_{0}}{\partial x_{0}}-\frac{\partial u_{1}}{\partial x_{1}}-\frac{\partial u_{2}}{\partial x_{2}}-\frac{\partial u_{3}}{\partial x_{3}}\right) d x y\right. \\
& +e_{1}\left(\frac{\partial u_{1}}{\partial y_{0}}+\frac{\partial u_{0}}{\partial y_{1}}+\frac{\partial u_{3}}{\partial y_{2}}-\frac{\partial u_{2}}{\partial y_{3}}+\frac{\partial u_{1}}{\partial x_{0}}+\frac{\partial u_{0}}{\partial x_{1}}+\frac{\partial u_{3}}{\partial x_{2}}-\frac{\partial u_{2}}{\partial x_{3}}\right) d x y \\
& +e_{2}\left(\frac{\partial u_{2}}{\partial y_{0}}-\frac{\partial u_{3}}{\partial y_{1}}+\frac{\partial u_{0}}{\partial y_{2}}+\frac{\partial u_{1}}{\partial y_{3}}+\frac{\partial u_{2}}{\partial x_{0}}-\frac{\partial u_{3}}{\partial x_{1}}+\frac{\partial u_{0}}{\partial x_{2}}+\frac{\partial u_{1}}{\partial x_{3}}\right) d x y \\
& \left.+e_{3}\left(\frac{\partial u_{3}}{\partial y_{0}}+\frac{\partial u_{2}}{\partial y_{1}}-\frac{\partial u_{1}}{\partial y_{2}}+\frac{\partial u_{0}}{\partial y_{3}}+\frac{\partial u_{3}}{\partial x_{0}}+\frac{\partial u_{2}}{\partial x_{1}}-\frac{\partial u_{1}}{\partial x_{2}}+\frac{\partial u_{0}}{\partial x_{3}}\right) d x y\right]
\end{aligned}
$$

By Equations (3.1) and (3.2), we have $d(\kappa F)=0$. By Stokes theorem, we have

$$
\int_{b G} \kappa F=\int_{G} d(\kappa F)=0 .
$$




\section{References}

[1] Futer, R., Über die analytische Darstellung der refulären Funktionen einer Quaternionen-variablen, Comment. Math. Helv. 8 (1935), 371-378.

[2] Gürsey, F. and Tze, H.C., Complex and Quaternionic Analyticity in Chiral and Gauge Theories I, Ann. of Physics 128 (1980), 29-130.

[3] Hamilton, W.R., Hamilton's discovery of quaternions, Math. Mag. 49 (1976), $227-234$

[4] Kajiwara, J., Li, X.D. and Shon, K.H., Regeneration in complex, Quaternion And Clifford analysis, Lecture Notes in Pure and Applied Mathematics. Marcel Dekker. Inc., 214 (2000), 287-298.

[5] Kajiwara, J., Li, X.D. and Shon, K.H., Function spaces in complex and Clifford analysis, Inhomogeneous Cauchy Riemann system of quaternion and Clifford analysis, Proc. the 14th International Conference on Finite or Infinite Dimensional Complex Analysis and Application. Hue. Viet Nam. Hue University 14 (2006), 127-155

[6] Oka, K., Sur les fonctions analytiques de plusieurs variables complexes. IV. Domaines d'holomorphie et domaines rationellement convexes, Jap. J. Math. 17 (1941), 517-521.

[7] Oka, K., Sur les fonctions analytiques de plusieurs variables complexes. II. Domaines d'holomorphie, J. Sci. Hiroshima Univ. 7 (1937), 115-130.

[8] Oka, K., Sur les fonctions analytiques de plusieurs variables complexes. III. Deuxième problème de Cousin, J. Sci. Hiroshima Univ. 9 (1939), 7-19.

[9] Oka, K., Sur les fonctions analytiques de plusieurs variables complexes. VI. Domaines pseudoconvexes, Tohoku Math. J. 49 (1942), 15-22.

[10] Oka, K., Sur les fonctions analytiques de plusieurs variables complexes. VII. Sur quelques notions arithmé tiques, Bull. Soc. Math. France 78 (1950), 1-27.

[11] Oka, K., Sur les fonctions analytiques de plusieurs variables complexes. IX. Domaines finis sans point critique intérieur, Jap. J. Math. 23 (1953), 97-155.

[12] Oka, K., Sur les fonctions analytiques de plusieurs variables complexes. I. Domaines convexes par rapport aux fonctions rationelles, J. Sci. Hiroshima Univ. 6 (1936), 245-255.

[13] Oka, K., Sur les fonctions analytiques de plusieurs variables complexes. V. L'intégrale de Cauchy, Jap. J. Math. 17 (1941), 525-531.

[14] Weil, A., L'intégrale de Cauchy et les fonctions de plusieurs variables, Math. Ann. 111 (1935), 178-182.

Hyun Sook Jung

Department of Mathematics, Pusan National University,

Busan 609-735, Korea.

E-mail: hsjung@pusan.ac.kr

$\mathrm{Su}$ Jin Ha

Department of Mathematics, Pusan National University, 
Busan 609-735, Korea.

E-mail: adorm84@naver.com

Kwang Ho Lee

Department of Mathematics, Pusan National University, Busan 609-735, Korea.

E-mail: kwangho1477@naver.com

Su Mi Lim

Department of Mathematics, Pusan National University, Busan 609-735, Korea.

E-mail: aiqiqiq@naver.com

Kwang Ho Shon

Department of Mathematics, Pusan National University, Busan 609-735, Korea.

E-mail: khshon@pusan.ac.kr 